Original Research Article

\title{
Diagnostic laparoscopy in right iliac fossa pain
}

\author{
Ravichandran K. S ${ }^{1}$, Sivachandran K. ${ }^{2}$ \\ ${ }^{1}$ Dr. Ravichandran K. S., Associate. Professor, ${ }^{2}$ Dr. Sivachandran K., Senior Resident, both authors are affiliated with \\ Department of General Surgery, Melmaruvathur Adhiparasakthi Institute of Medical Science and Research, \\ Melmaruvathur, Tamilnadu, India.
}

Corresponding Address: Dr. Ravichandran K. S, Associate. Professor, Department of General Surgery, Melmaruvathur Adhiparasakthi Institute of Medical Science and Research, Melmaruvathur, Tamilnadu, India. Email: drksravichandranms@yahoo.com

\begin{abstract}
Background: Abdominal pain is a common problem in both males and females. Early Diagnosis is needed to rule out life threatening emergencies. Diagnostic laparoscopy is very much useful in giving proper treatment and avoiding negative laparotomies. Aims \& objectives: To study the use of laparoscope in patients with acute or chronic right iliac fossa pain, to diagnose and confirm conditions like acute appendicitis, abscess, perforation, mass formation, etc. where clinical and imaging studies are inconclusive. Materials \& Methods: Place: Department of General surgery, Melmaruvathur Adhiparasakthi Institute of Medical Science and Research, Melmaruvathur. Period of study: June 2017 to January 2018. Material: All patients with right iliac fossa pain in the ages between ten years and seventy years were included in this study. Results: Total 50 patients were included in this prospectivestudy. 29 patients (58\%) were found to have acute appendicitis. Complicated appendix cases like mass formation, perforated appendix, appendicular abscess was found in 12 patients (24\%). Normal looking appendix was found in 3 patients $(6 \%)$. Non appendix lesions were found in 6 patients (12\%). Conclusions: The best approach in right iliac fossa pain is to do diagnostic laparoscopy and proceed, rather than going for open appendisectomy. Diagnostic laparoscopy gives all benefits of minimal invasive surgery. Not much of pain, shorter period of hospitalization, small scars, low infection rates and most importantly, accurate diagnosis and the correct treatment of most of the intra abdominal conditions are the gifted things.
\end{abstract}

Keywords: Diagnostic laparoscopy, Appendicectomy, Appendicitis, Right iliac fossa pain

\section{Introduction}

Right iliac fossa pain is a common problem among children and female. Acute appendicitis is a common abdominal emergency which requires immediate surgery. Diagnostic laparoscopy gives many advantages in the management of many intra-abdominal conditions wherethe correct diagnosis could not be established clinically or even withthe help of imaging studies. (Acute appendicitis, pelvicin flammatory disease, Hollow viscus perforation, bowelischaemia etc.) [1,2].

In most of the patients, the clinical signsand symptoms are masked by the treatments given by the different physicians at different hospitals at different points of time. Different radiologists giving different reports of imaging studies and advising to correlate clinically. In these circumstances, there is an absolute need to search

Manuscript Received: $4^{\text {th }}$ November 2018

Reviewed: $14^{\text {th }}$ November 2018

Author Corrected: $20^{\text {th }}$ November 2018

Accepted for Publication: 23 ${ }^{\text {rd }}$ November 2018 for an alternate diagnostic tool. Here we thought of diagnostic laparoscopyto solve the issue. Diagnostic laparoscopy reduces the number of negative laparotomies in acute abdomen and prevents severe peritonitis which may occur as a result of delay in diagnosis $[3,4,5]$.

\section{Materials \& Methods}

Place of study: Department of general surgery. Melmaruvathur Adhiparasakthi institute of medical sciences. Melmaruvathur. Tamilnadu

Type of Study: Prospective study

Criteria: Patients in the age group 10-70 years and both male and female with acute or chronic right iliac fossa pain and suspected appendicitis were subjected to diagnostic laparoscopy, after proper consent and proper pre operative workup. 


\section{Original Research Article}

Exclusion criteria: Patients with suspicion of malignancy, severe co morbid illness, pulmonary and cardiac disorders, were excluded from the study. Patients with age less than 10 and more than 70 are were also excluded from the study.

Methods- All lesions which were diagnosed, were managed as per the standard protocol. For all patients, Post operative pain, Reintroduction of diet, hospital stay, pre operative and post operative complications were properly evaluated. All patients were followed up for up to 3 months.

Procedure- Diagnostic laparoscopy was performed with proper care. Ryle's tube used to decompress thestomach and Foleys catheter to empty the urinary bladder. Antibiotics were started pre operatively and continued according to the findings. Pneumoperitoneum was created by using direct access method. Intraabdominal pressure was kept initially 12 to $14 \mathrm{mmHg}$ which was reduced to $10 \mathrm{~mm} \mathrm{Hg}$ after insertion of all trocars.

First $10 \mathrm{~mm}$ trocar was put in supra umbilical position. 30 degree telescope (Stryker, USA) was used and further trocars, $10 \mathrm{~mm}, 5 \mathrm{~mm}$ were inserted depending on the case. Most common trocar positions used were two
$5 \mathrm{~mm}$ trocars in supra pubic and left iliac fossa. In some cases $10 \mathrm{~mm}$ trocar was used in left iliac fossa. Foot end of the patient was elevated with left tilt in suspected cases of appendicitis. Appendicectomy was done when appendix was found to be inflamed. A drain was kept in case of appendicular abscess.

Normal looking appendix with no obvious any other pathology was found in 3 cases. In these cases also, appendix was removed for histopathological examination. Other pathologies were dealt accordingly. Conversion to midline laparotomy was done in two cases.

Open appendisectomy was done in 3 cases. In case of spillage of pus, blood or purulent fluid, suction irrigation with normal saline was done. Drains were kept in selected cases and the Drain was removed after 48 to 72 hours.

Orals were allowed after 12 to 48 hours depending on pathology. Patients were discharged after 3 to 7 days. All specimens were sent for histopathological examination.

Follow up of the patients was done after one week, two weeks and after onemonth.

\section{Results}

50 patients underwent diagnostic laparoscopy. 22 patients were male and 28 patients were female. Most of the patients were in age group 20 to 40 yrs. Conversion to laparotomy was done in two cases.

Table 1: Per operative findings

\begin{tabular}{|c|c|c|c|c|}
\hline S.No & Diagnosis & Total, no. of males & $\begin{array}{c}\text { Total No. of } \\
\text { Females }\end{array}$ & Total No of Patients \\
\hline 1. & Acute appendicitis & 10 & 19 & $29(58 \%)$ \\
\hline 2. & Appendicular abscess & 3 & 2 & $5(10 \%)$ \\
\hline 3. & Appendicular perforation & 2 & 2 & $3(6 \%)$ \\
\hline 4. & Appendicular lump & 2 & 1 & $4(8 \%)$ \\
\hline 5. & Normal lookingappendix & 2 & 1 & $3(6 \%)$ \\
\hline 6. & Ectopic pregnancy & 0 & 0 & $1(2 \%)$ \\
\hline 7. & Iliocaecal tuberculosis & 1 & 2 & $2(2 \%)$ \\
\hline 8. & Ovarian cyst & 0 & 0 & $1(2 \%)$ \\
\hline 9. & Small bowel intussusception & 1 & 0 & $1(2 \%)$ \\
\hline 10. & Perforated ileum & 1 & 28 & 50 \\
\hline
\end{tabular}

Acute appendicitis \& Appendicular abscess are two most common causes of right iliac fossa mass. 


\section{Original Research Article}

\section{Discussion}

Abdominal pain is the most common complaint of majority of the patients attending surgical clinics. There are many number of diseases and issues including psychosomatic disorders, which can cause abdominal pain. Many patients take self remedies and the pain scenario changes a lot. Sometimes, it becomes very difficult to elicit proper history. Intensity of pain and pain threshold also varies considerably from individual to individual. Increasing incidence of medico legal cases threaten the treating doctor to come to clearcut diagnosis for the sick person. If not diagnosed correctly, the patient will be suffering a lot. Even imaging studies cannot help in diagnosing conditions like bowel ischemia. Thus, the surgeon may be at a great difficulty.

Abdomen is Pandoras box. The standard teaching is whenever in doubt, always do a laprotomy and open the abdomen and, see the things. But now, with laparoscopy, things are changing laparoscopy is an excellent tool, when ever the diagnosis is in doubt.It is far better than open laprotomy.

Use of laparoscopy in the management of acute as well as chronic abdominal pain is well established in literature [1,2,4,5,6,7]. Correct diagnosis and best treatment are possible by laparoscopy in most of the abdominal emergencies [1,5.8]. It is the best option in children and young female due to multiple differential diagnoses. $[9,10]$.

A review of the published research papers on Appendicitis shows the following. Laparoscopic appendectomy should be recommended as standard procedure for acute appendicitis [11]. Laparoscopy is an efficient diagnostic and treatment tool in children with chronic unexplained abdominal pain, it avoids serial examinations, prolong admission, battery of investigations and unnecessary surgeries [12].

Laparoscopy may aid in the diagnosis of acute right iliac fossa pain. However, intra operative diagnosis is not easy with almost one- third of apparently normal appendices being inflamed histologically. We wound therefore advocate the removal of a normal looking appendix in the absence of other explanatory pathology $[13,14]$. Laparoscopic removal of the normal appendix produces no added morbidity or increase in length of hospitalization as compared to diagnostic laproscopy. It demonstrates cost effectiveness by preventing missed and future appendicitis. Incidental laparoscopic appendecetomy is the preferred treatment option [15].
Due to the consistently false negative rate of DL, and the low morbidity rate for laparoscopic appendectomy, we support incidental appendectomy in patients with lower abdominal pain [16].

All women of child bearing age suspected of having acute appendicitis should undergo diagnostic laparoscopy prior to the planned appendicectomy, regardless of the certainty of the preoperative diagnosis.

This is currently the only way to reduce the negative appendicectomy rate and establish a correct diagnosis allowing prompt and appropriate treatment. In male patients and postmenopausal women one may proceed directly to emergency appendicectomy [17].

Laparoscopic appendicectomy is increasingly being performed. Laparoscopy is often used as a diagnostic tool in general surgical patients, particularly in women, with lower abdominal pain. Most of the women patients are undergoing diagnostic laparoscopy, with or without appendectomy. This has resulted in a lower positive appendectomy rate, but a higher yield of a diagnoses other than appendicitis, in the laparoscopic group, overall appendectomy rates, however, have remained unchanged [18].

In our study, out of 50 patients withright iliac fossa pain, 41 patients had appendicular pathology, 3 patients had normal looking appendix with no other abdominal findings and remaining 6 patients had non appendicular pathology.

Laparoscopic appendesectomy was done in 26 patients having acute appendicitis. Open Appendisectomy was done in 3 patients with acute appendicitis, reasons being technical. Appendisectomy was done as per standard protocol. Allthe specimens were sent for histopathological examination. The Appendicular abscess patient was treated with drainage and was put on drain.

Appendicular lumps were left untouched. In our series of patients, 3 patients had appendicular perforation. Laparoscopic appendectomy with thorough peritoneal lavage was done in these three patients. Pus \& peritoneal fluid was sent for microbiology. Three patients had normal looking appendix. These three patients were also done with Appendisectomy. Overall total of 35 patients out of 50 had Appendisectomy. 32 laparoscopic Appendisectomy and 3 open appendisectomy. 


\section{Original Research Article}

\section{Conclusion}

Diagnostic laparoscopy followed by appendisectomy should be should be the standard approach for appendicitis, irrespective of its anatomical and pathological types. It is the method of choice for children and young women and obese patients.

It reduces rate of negative laparotomies.

Thorough exploration of peritoneal cavity is possible with laprosocpy. Non appendiceal lesions can also be diagnosed and treated properly.

Smallincision, Small scar, Minimal complications like wound infection, postoperative adhesions, incisional hernia, loss of fertility are some of the real benefits of laparoscopy. Above all, Diagnostic laparoscopy has a sensitivity and specificity of $100 \%$.

Acknowledgement: I thank Dr Sivachandran for his contribution during this studyand also in the process of manuscript preparation.

Conflict of interest: None declared.

Funding: Nil, Permission from IRB: Yes

\section{References}

1. Karamanakos SN, Sdralis E, Panagiotopoulos S, et al. Laparoscopy in the emergency setting: a retrospective review of 540 patients with acute abdominal pain. Surg LaparoscEndoscPercutan Tech. 2010 Apr;20(2):119-24. doi: 10.1097/SLE.0b013e3181d87178.

2. Ning N, Xia SY, Ma B, et al. [Application of laparoscopic technique in acute abdomen of gastrointestinal surgery]. Zhonghua Wei Chang Wai Ke Za Zhi. 2013 Oct;16(10):960-2.

3. Drăghici I, Drăghici L, Popescu M, et al. Laparoscopic exploration in pediatric surgery emergencies. J Med Life. 2010 Jan-Mar; 3 (1):90-5.

4. Sauerland S, Agresta F, Bergamaschi R, et al. Laparoscopy for abdominal emergencies: evidencebased guidelines of the European Association for Endoscopic Surgery. Surg Endosc. 2006 Jan; 20 (1):1429. Epub 2005 Oct 24. DOI: 10. 1007/ s00464-0050564-0

5. Agrusa A, Romano G, Di Buono G, et al. Laparoscopic approach in abdominal emergencies: a 5-year experience at a single center. G Chir. 2012 Nov-Dec; 33 (11-12):400-3.
6. Reiertsen O, Trondsen E, Bakka A, Andersen OK, LarsenS, Rosseland AR. Prospective nonrandomized study of conventional versus laparoscopic appendectomy. World J Surg. 1994; 18:411-415. (Pub Med)

7. Gajalakshmi CK, Krishnamurthi S, Ananth R, et al. Cervical cancer screening in Tamilnadu, India: a feasibility study of training the village health nurse. Cancer Causes Control. 1996 Sep;7(5):520-4.

8. Golash V, Willson PD. Early laparoscopy as a routine procedure in the management of acute abdominal pain: a review of 1,320 patients. Surg Endosc. 2005 Jul;19 (7): 882-5. Epub 2005 May 12.DOI:10.1007/s00464004-8866-1

9. Gaitán HG, Reveiz L, Farquhar C, et al. Laparoscopy for the management of acute lower abdominal pain in women of childbearing age. Cochrane Database Syst Rev. 2014 May 22; (5):CD007683. doi: 10.1002 /1465 1858. CD007683.pub3.

10. Lobe ET. Laparoscopic surgery in children. Currprobl. Surg. 1998: 35: 869-948. (Pub Med)

11. Parteckev L et al. Appendicitis : A pro for laparoscopic appendectomy as the standard procedure for acute appendicitis. Langenbecks Arch Surg. 2010 Nov; 395(8): 1069-76. Dol:10.1007/s00423-009-05678. Epub 2009 Nov 19.

12. Talat N, Afzal M, Ahmad S, et al. Role of diagnostic laparoscopy in evaluation and treatment of chronic abdominal pain in children. J Ayub Med Coll Abbottabad. 2016 Jan-Mar;28(1):35-8.

13. van den Broek WT, Bijnen AB, van Eerten PV, et al. Selective use of diagnostic laparoscopy in patients with suspected appendicitis. Surg Endosc. 2000 Oct;14 (10): 938-41.

14. Phillips AW, Jones AE, Sargen K. Should the macroscopically normal appendix be removed during laparoscopy for acute right iliac fossa pain when no other explanatory pathology is found? Surg Laparosc Endosc Percutan Tech. 2009 Oct;19(5):392-4. doi: 10. 1097/SLE.0b013e3181b71957.

15. Greason KL, Rappold JF, Liberman MA. Incidental laparoscopic appendectomy for acute right lower quadrant abdominal pain. Its time has come. Surg Endosc. 1998 Mar;12(3):223-5. 


\section{Original Research Article}

16. Chiarugi M, Buccianti P, Decanini L, et al. What you see is not what you get". A plea to remove a 'normal' appendix during diagnostic laparoscopy. Acta Chir Belg. 2001 Sep-Oct;101(5):243-5.

17. Borgstein PJ, Gordijn RV, Eijsbouts QA, et al. Acute appendicitis--a clear-cut case in men, a guessing game in young women. A prospective study on the role of laparoscopy. Surg Endosc. 1997 Sep;11(9):923-7.

18. Akbar F, Yousuf M, Morgan RJ, et al. Changing management of suspected appendicitis in the laparoscopic era. Ann R Coll Surg Engl. 2010 Jan; 92 (1): 65-8. doi: 10.1308/003588410X1251883 6439920 .

\section{How to cite this article?}

Ravichandran K. S, Sivachandran K. Diagnostic laparoscopy in right iliac fossa pain. Surgical Update: Int J surg Orthopedics.2018;4(4):139-143.doi:10.17511/ijoso.2018.104.02. 